\title{
Respiratory-phase Domain Analysis of Heart Rate Variability Can Accurately Estimate Cardiac Vagal Activity during a Mental Arithmetic Task
}

\author{
K. Kotani ${ }^{1}$, M. Tachibana' ${ }^{2}$ K. Takamasu ${ }^{2}$ \\ 'Graduate School of Information Science and Technology, The University of Tokyo, Tokyo, Japan \\ ${ }^{2}$ Graduate School of Engineering, The University of Tokyo, Tokyo, Japan
}

\begin{abstract}
Summary
Objectives: The objectives of this paper were to present a method to extract the amplitude of RSA in the respiratory-phase domain, to compare that with subjective or objective indices of the MWL (mental workload), and to compare that with a conventional frequency analysis in terms of its accuracy during a mental arithmetic task.

Methods: HRV (heart rate variability), ILV (instantaneous lung volume), and motion of the throat were measured under a mental arithmetic experiment and subjective and objective indices were also obtained. The amplitude of RSA was extracted in the respiratoryphase domain, and its correlation with the load level was compared with the results of the frequency domain analysis, which is the standard analysis of the HRV.

Results: The subjective and objective indices decreased as the load level increased, showing that the experimental protocol was appropriate. Then, the amplitude of RSA in the respiratory-phase domain also decreased with the increase in the load level. The results of the correlation analysis showed that the respiratory-phase domain analysis has higher negative correlations, -0.84 and -0.82 , with the load level as determined by simple correlation and rank correlation, respectively, than does frequency analysis, for which the correlations were found to be -0.54 and -0.63 , respectively. In addition, it was demonstrated that the proposed method could be applied to the short-term extraction of RSA amplitude.

Conclusions: We proposed a simple and effective method to extract the amplitude of the respiratory sinus arrhythmia (RSA) in the respiratory-phase domain and the results show that this method can estimate cardiac vagal activity more accurately than frequency analysis.
\end{abstract}

\section{Keywords}

Heart rate variability, Hilbert transform, respiratory phase, respiratory sinus arrhythmia

Methods Inf Med 2007; 46: 376-385

doi:10.1160/ME0352

\section{Introduction}

Heart rate variability (HRV) has been well studied because of its physiological, psychophysiological and medical importance $[1,2]$. The conventional methods of analyzing HRV are time domain (e.g., mean, standard deviation, and histogram) and frequency domain analysis. A frequency of more than $0.15 \mathrm{~Hz}$ in the frequency domain is called the HF (high-frequency) component $[1,3]$, which is respiratory modulation of the heart rate and is known physiologically as respiratory sinus arrhythmia (RSA). Based on the results of an animal experiment [4] and a pharmacological blockade experiment involving humans [5], the amplitude of RSA has been regarded as a selective index of cardiac vagal activity. RSA can be obtained non-invasively and provides a measure of cardiac vagal activity apart from sympathetic nervous activity. Accurate extraction of RSA is, therefore, useful in various fields. From the psychophysiological and ergonomic point of view, the amplitude of RSA is used for estimating mental workload (MWL) [2, 6]. The estimation of MWL is important for comfortable manmachine interface and for the improvement of the workers' environment. From the physiological and medical point of view, RSA represents the large fluctuation of HRV that is mediated by cardiac vagal activity. It is very important to clarify these cardiovascular dynamics. Further investigation of these dynamics and their conversion during pathological states is needed. Furthermore, evaluating RSA during work is also important in preventive medicine, because autonomic nervous activity affects immunity [7].
For the more accurate extraction of RSA, combining advanced signal processing with physiological insight seems to be essential because HRV has 'complex' features [1,8, 9]. In the present study, we proposed a simple and effective method for the accurate extraction of the amplitude of RSA based on the concept of respiratory-phase domain analysis [10]. The objectives of this study were to present a method for extracting the amplitude of RSA in the respiratory-phase domain, to compare that method with subjective or objective indices of the MWL, and to compare the method with the conventional frequency analysis in terms of its accuracy during a mental arithmetic task.

\section{Methods}

\subsection{Experimental Procedures}

Seven healthy males (with ages ranging from 21 to 27 years) were studied at rest in the sitting posture. During a mental arithmetic task, three main categories of MWL were measured [11], namely, subjective, objective, and physiological indices. The mental arithmetic task was as follows: Two integers from 1 to 49 were displayed on the screen, and the subjects were asked to give the sum of these values within a given time limit. In order for the subjects to be able to perform the response only by one action of the finger, they were further asked to calculate the sum of the number in the ones place and the number in the tens place in the answer, and input the number in the ones place from the answer with keyboard in hand. The experimental conditions were as 
shown in Figure 1. The session-length of each task was $300 \mathrm{~s}$, which matches the length of the general short-term recordings of HRV [1]. The rest condition was also analyzed during the $300 \mathrm{~s}$ by discarding the first $60 \mathrm{~s}$. The load level was increased by gradually shortening the intervals between the questions. As a subjective index, the NASAtask load index (NASA-TLX) [12] was performed twice in the experiment and the weighted workload was calculated. NASATLX is a method of subjective task evaluation that involves classifying the degree and importance of six factors of the workload. As an objective index, the correct answer rate was calculated. As a physiological measurement, R-R intervals (RRI) by electrocardiogram (AC-601, NihonKoden), instantaneous lung volume (ILV) by elastic chest band (TR-755T, NihonKoden), and the motion of the laryngeal prominence by an accelerometer (8304B2, Kistler) were recorded continuously. The electrocardiogram was digitized at a sampling frequency of $1000 \mathrm{~Hz}$, while ILV and the motion of the laryngeal prominence were digitized at a sampling frequency of $100 \mathrm{~Hz}$.

\subsection{Data Analysis}

The RSA was extracted by respiratoryphase domain and frequency domain. The procedures of respiratory-phase domain analysis and frequency domain analysis are described below.

\subsubsection{Respiratory-phase Domain Extraction of RSA}

A schematic diagram of the proposed signal-processing algorithm (i.e. respiratoryphase domain extraction of the RSA amplitude) is shown in Figure 2. The proposed method was composed of four parts (interpolation of discrete RRI, determining the respiratory phase, selecting the data, and ensemble averaging). The method of signal processing regarding each box in the diagram is described below.

In the box labeled DCSI + inverse, discrete RRI is interpolated using the derivative of cubic spline interpolation (DCSI)

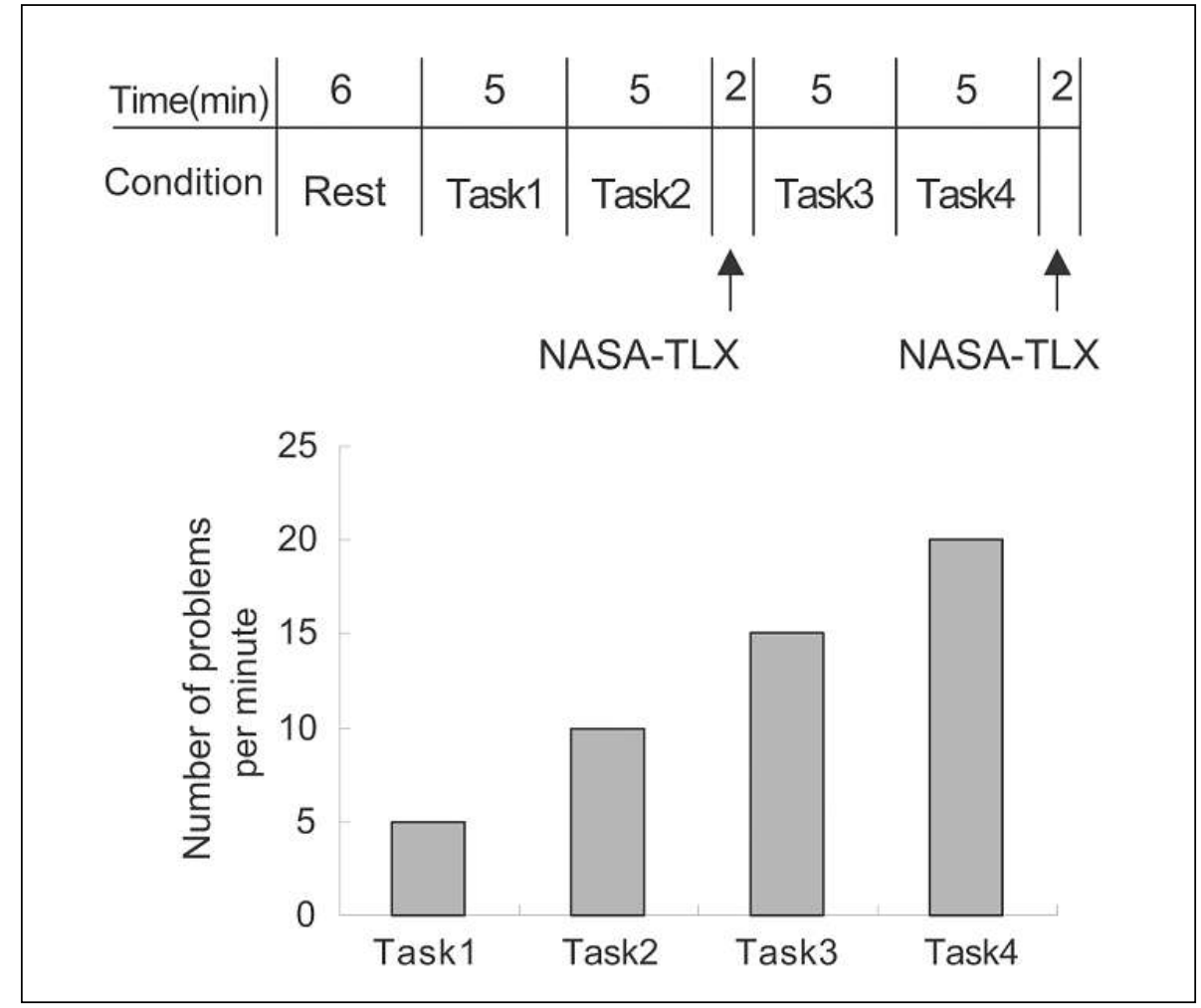

Fig. 1 Experimental condition. The number of problems per minute increased linearly.

method [13] in order to obtain an instantaneous heart rate that has no time delay. The DCSI method is based on the integral pulse frequency modulator (IPFM) model:

$M\left(t_{k}\right)=\int_{t_{0}}^{t_{k}} m(t) d t=k$

where $t_{k}(k=0,1,2, \ldots, n)$ are the times at which heartbeats were observed and $m(t)$ is the instantaneous heart rate. In the DCSI method, $m(t)$ is obtained from the first derivative of the interpolated $M\left(t_{k}\right)$ using a cubic spline function. The inverse of this function $m(t)$ was used as instantaneous RRI. The no time delay feature is very important for the proposed method because the delay between interpolated RRI and the respiratory phase is variable even if the delay is constant in the time domain.

From the BPF and Hilbert transform boxes, the instantaneous respiratory phase was calculated after filtering. The raw ILV data contained low-frequency trends and electrical noise, both of which disguised the calculation of the respiratory phase. Therefore, raw ILV data were passed through a linear phase finite impulse response filter whose pass-band was $0.1-10.0 \mathrm{~Hz}$. Here, the high frequency limitation was large in value compared with the respiratory frequency so as not to lose the harmonics of the respiratory oscillation. An instantaneous respiratory phase was estimated using an analytic signal. The analytic signal is a complex function of time defined by

$\psi(t)=s(t)+j \widetilde{s}(t)=A(t) e^{j \phi(t)}$

where $j$ is the imaginary unit, $s(t)$ is the ILV data in this case, and the function $s(t)$ is the Hilbert transform of $s(t)$,

$\tilde{s}(t)=\pi^{-1} \mathrm{P} . \mathrm{V} \cdot \int_{-\infty}^{\infty} \frac{s(\tau)}{t-\tau} d \tau$

where $\tau$ is the variable for integration and P.V. means that the integral is taken in the sense of the Cauchy principal value [14]. The instantaneous phase $\phi(t)$ of the signal $s(t)$ was thus uniquely defined from Equation 2. Finally, the instantaneous RRI, 


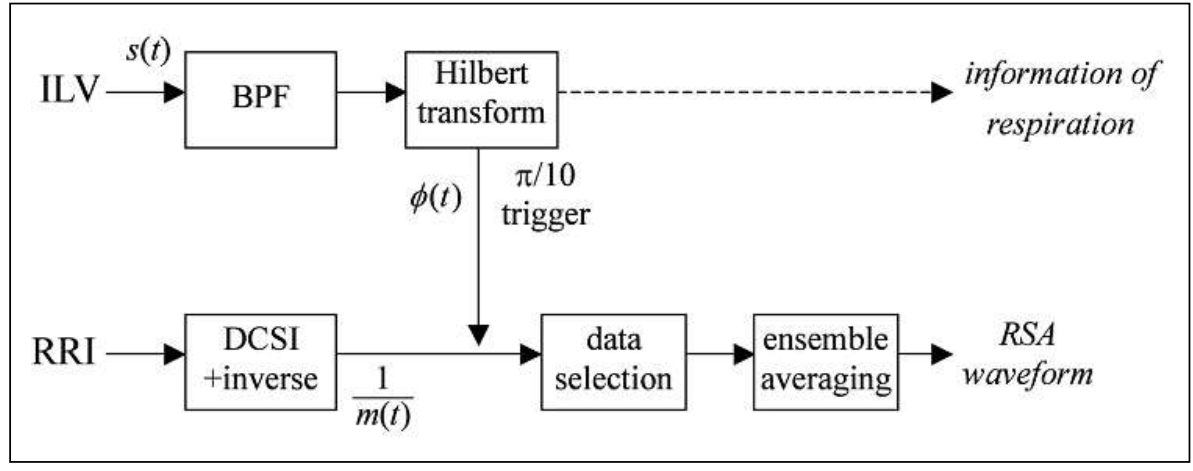

Fig. 2 Schematic diagram of the signal-processing method for extracting the RSA waveform using the respiratory-phase domain analysis. The BPF box refers to band-pass filtering.

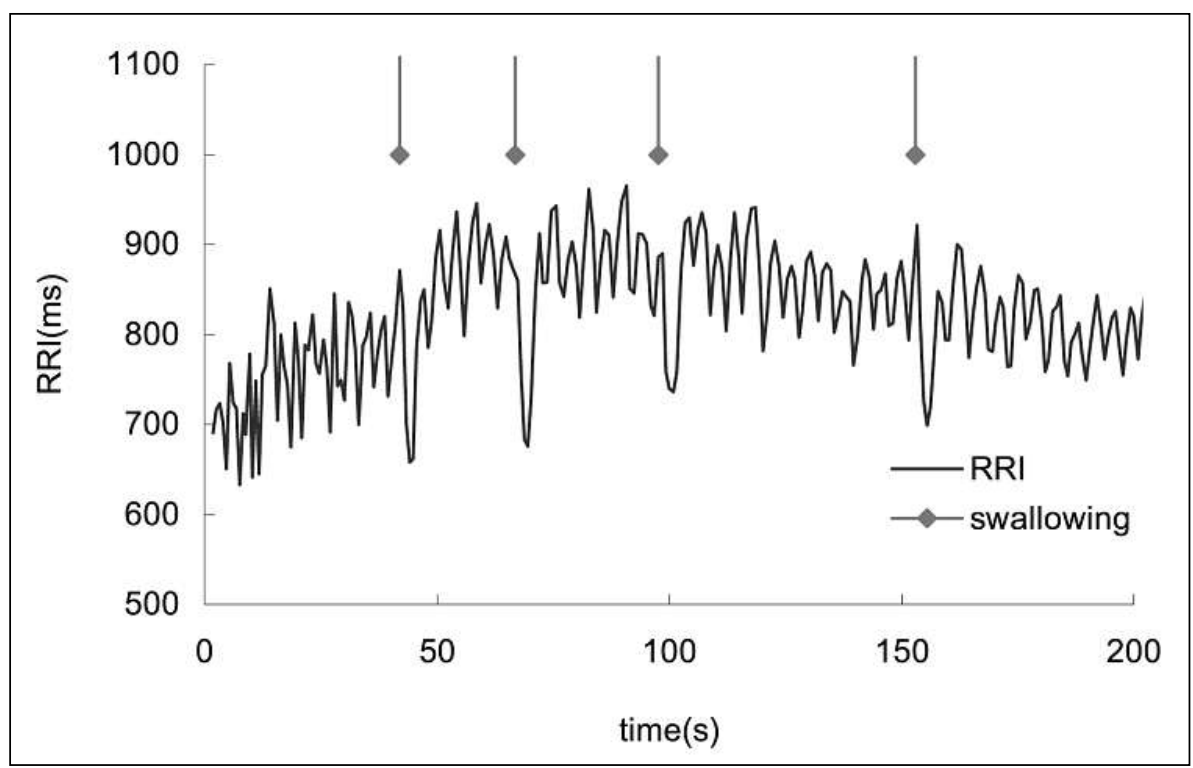

Fig. 3 RRI time series and the occurrence of swallowing. Following the swallowing, temporary tachycardia was observed.

$1 / m(t)$, was re-sampled at every $\pi / 10 \mathrm{rad}$ of the respiratory phase, yielding an equidistantly sampled RRI variability.

In the data selection box, the data that did not reflect vagal activity were removed. In the case of low-frequency respiration less than $0.15 \mathrm{~Hz}$, RSA was mixed with or entrained with the oscillation caused by blood pressure, which is called Mayer wave-related sinus arrhythmia (MWSA). Similarly in low-frequency respiration, the pulmonary stretch receptor inhibits the activity of the inspiratory neurons by the HeringBreuer reflex. This effect causes the separation of the two vagal reduction effects (the pulmonary stretch receptor inhibits vagal activity, but the inspiratory neurons do not) and induces a gap between the RSA phase and the respiratory phase [10]. Therefore, the data containing low-frequency respiration of less than $0.15 \mathrm{~Hz}$ were removed. Overly rapid respiration in which the difference in the continuous respiratory phase exceeded $\pi / 10$ rad was also removed. In addition, the authors found that swallowing induced tachycardia which might be caused by the sudden change of intrathoracic pressure and that this tachycardia disappears within one respiration, which can be clearly observed in Figure 3. The time at which the swallowing began was detected by an accelerometer and the data containing the respiration during swallowing were removed.

Finally, in the ensemble averaging box, the mean RSA waveform was calculated, and the amplitude of RSA was determined. Figure 4 shows the procedure that occurs in this box. The RRI variability is ensemble-averaged over the entire respiratory cycle $(2 \pi \mathrm{rad})$ in order to extract the mean RSA waveforms. RSA amplitude was calculated by the subtracting RSA in $2 \pi$ rad from RSA in $\pi$ rad. Here, it is assumed that the delay of the vagal neurotransmitter was negligible because it is well known that the vagal neurotransmitter is very fast. Under this assumption, the procedure is identical to the subtraction of the minimum value of the RSA waveform from the maximum.

\subsubsection{Frequency Domain Extraction of RSA}

For the frequency domain extraction of RSA, the standard method, Fast Fourier Transform (FFT), was chosen. Discrete RRI was interpolated by the inverse of the DCSI method and resampled at the frequency of 4 $\mathrm{Hz}$, and Bingham's cosine-shaped window was used as the window function. A power of $0.15-0.5 \mathrm{~Hz}$ of the FFT was calculated as the power of the RSA. Though the power of FFT was sometimes converted to another unit such as the logarithm of its power [2], we simply used the power because it is the standard unit of analysis $[1,15,16]$. The results were compared with the RSA amplitude determined by the respiratory-phase domain analysis.

\subsubsection{Statistical Analyses}

Two statistical analyses were performed. One analysis involved confirming whether the three indices followed the level of the load; the data for the weak task (Task 2) and strong task (Task 4) were compared by paired t-test in each index. The other analysis involved determining which analysis of the respiratory-phase domain or frequency domain followed the load more accurately. Here, the number of questions per minute was defined as the level of the load. This comparison was performed by two methods, simple correlation and rank correlation. At 


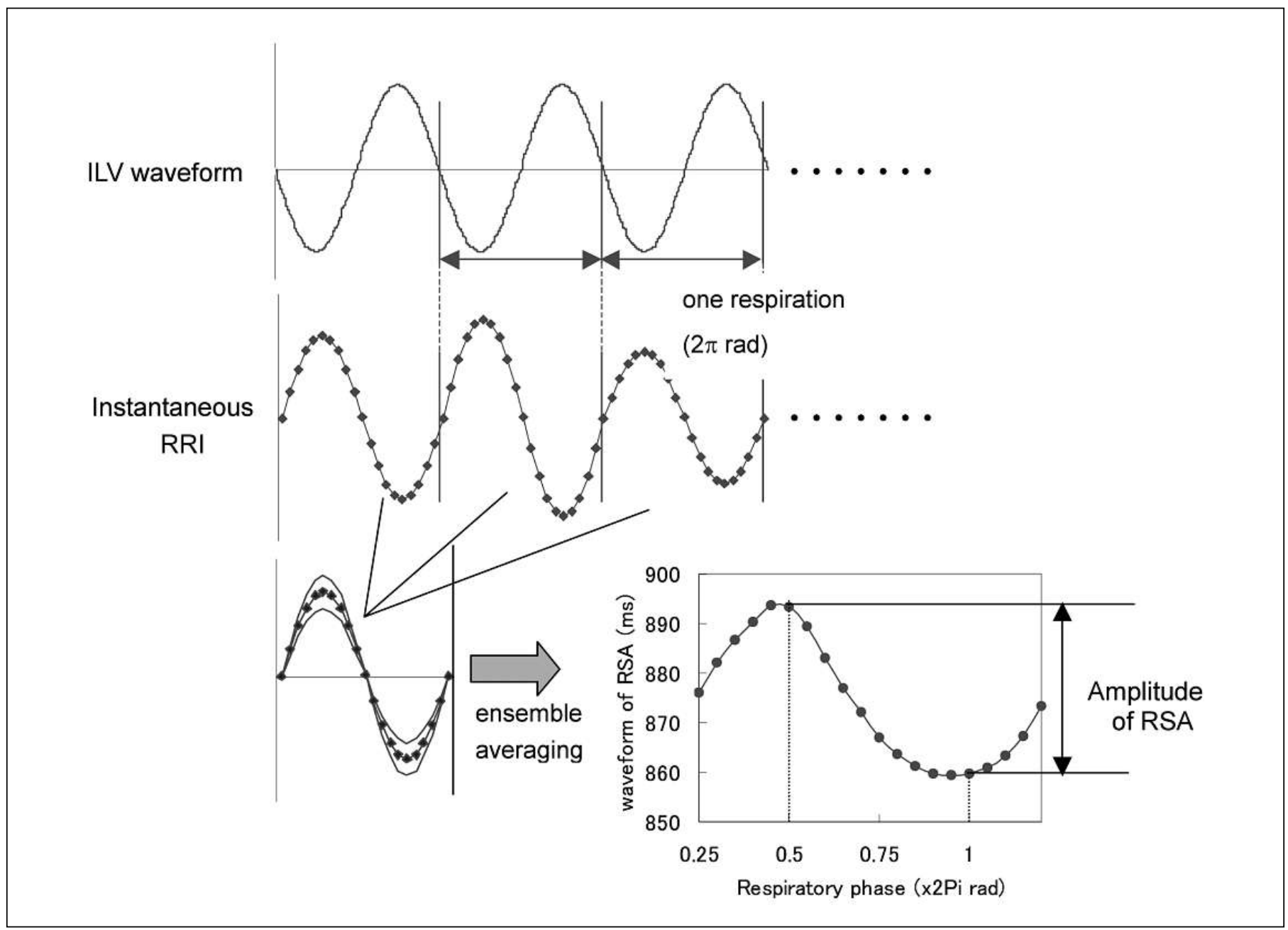

Fig. 4 Schematic graph showing the extraction of the mean RSA waveform and the calculation of RSA amplitude. The mean RSA waveform was obtained by ensemble averaging of the instantaneous RRI in the same phase. The amplitude of RSA is determined by subtracting

the RSA at the phase of $2 \pi$ rad from that at the phase of $\pi$ rad in the mean RSA waveform. In this figure, ILV is replaced by a periodic function for the sake of simplification. first, supposing that the RSA's amplitude (or power) responded linearly to the level of the load, the difference of the correlation between the load level and the RSA's amplitude (or power) was checked by the paired $\mathrm{t}$-test. Secondly, not supposing a linear response, the difference of the rank correlation between the load level and the RSA's amplitude was also checked by the paired t-test. Furthermore, because the paired t-test assumes a normal distribution of the data of the correlation coefficient, the Wilcoxon signed-rank test was also performed to confirm the results of the paired t-test. In both correlation analyses, it was expected that the stronger the load, the smaller the RSA's amplitude would be. Thus, if the correlation coefficient was near -1 , it could be regarded as an accurate index.

\section{Results}

\subsection{Comparison of the \\ Physiological Index with Subjective and Objective Index}

Figure 5 shows the results of the subjective, objective, and physiological indices of the experiment. The NASA-TLX score increased between Task 2 and Task 4 with statistical significance $(P<0.01)$. This re- sult indicates that the step of the load level is recognizable for subjects and is thus appropriate. The correct answer rate and the amplitude of RSA decreased as the task continued. Three indices in Task 2 and Task 4 that differed statistically in the subjective evaluation were focused and compared. It was found that the correct answer rate showed a statistically significant difference $(P<0.05)$. A large standard deviation of the correct answer rate was seen in Task 4, and seems to have been caused by individual computing ability. The amplitude of RSA also showed a statistically significant difference $(P<0.01)$. 
(a)

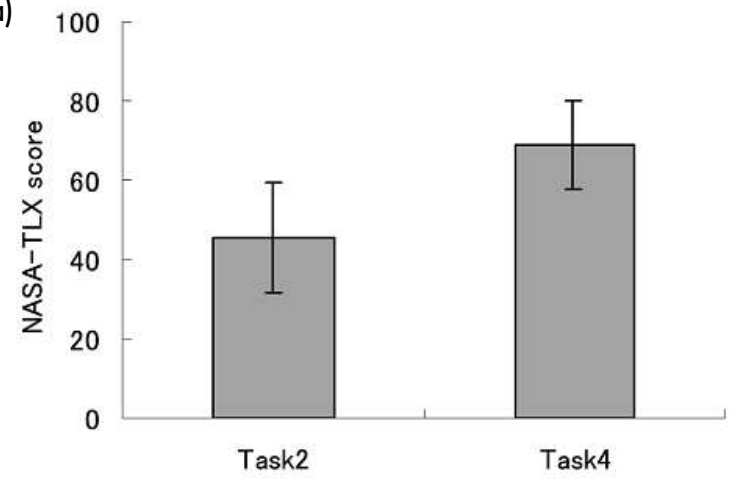

(c)

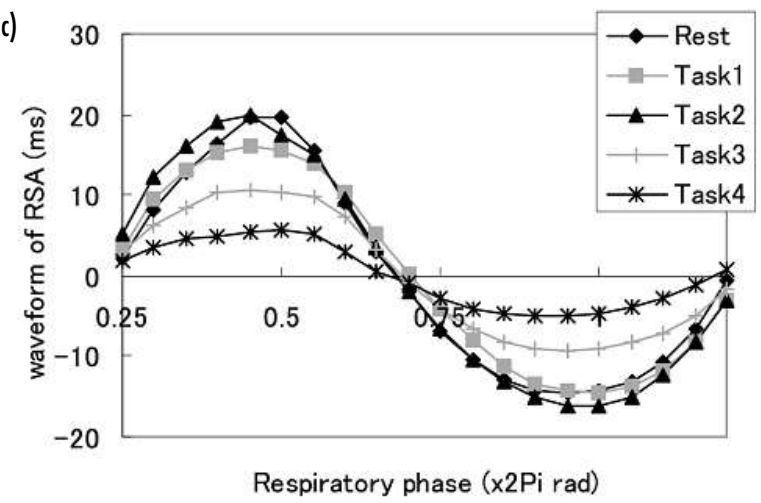

(b)

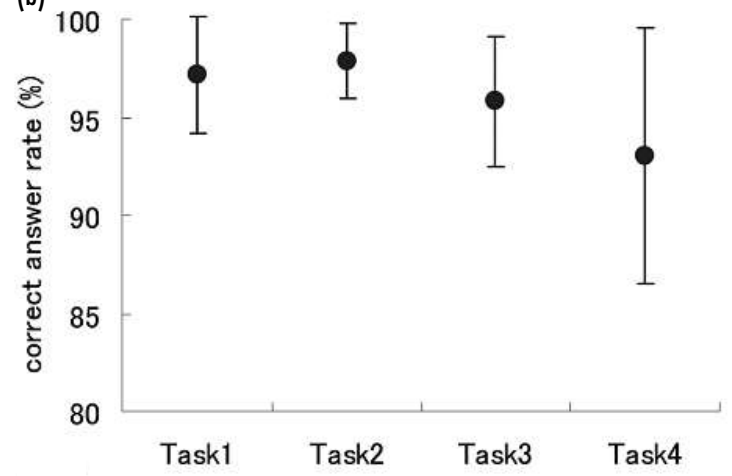

(d)

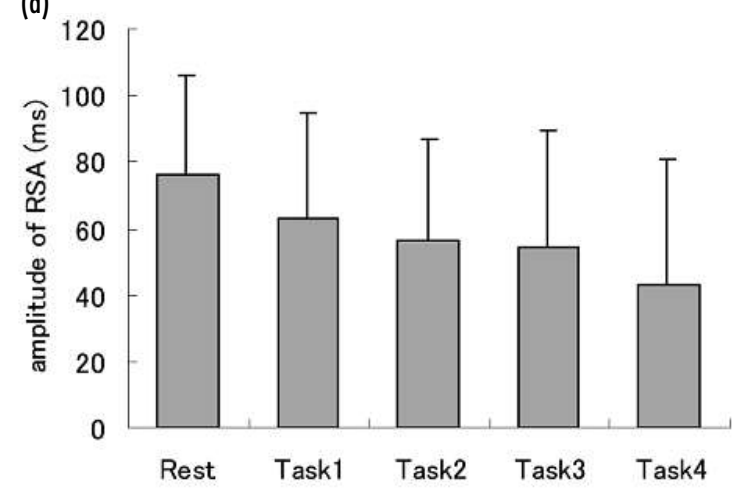

Fig. 5 Results of subjective, objective, and physiological indices of the experiment: (a) subjective index: NASA-TLX score, (b) objective index: correct answer rate, (c) representative waveform of the RSA in each task, and (d) physiological index: amplitude of RSA determined by respiratory-phase domain analysis. Data are mean values, and error bars show the standard deviation.

\subsection{Comparison with Conventional Frequency Analysis}

The amplitudes of RSA as determined by respiratory-phase domain and frequency domain were compared. Figure 6 shows the mean value (Fig. 6A) and results for the two subjects that had different properties; one had a large response (Fig. 6B) and the other had a small response (Fig. 6C) against the task. Figure $6 \mathrm{~B}$ shows the same tendency between respiratory-phase domain and frequency domain analysis, and Figure 6C does not. Table 1 shows the results of the two types of correlation coefficient, the simple correlation and the rank correlation, against the load level. In both cases, the respiratoryphase domain analysis had a higher negative correlation than did the frequency analysis (both having $P<0.05$ by paired t-test). The group-wise results of the large response and small response groups are also shown in Table 1. The respiratory-phase domain analysis of the five subjects who had small responses to the load showed larger negative correlations in comparison with the frequency analysis, while that of the two subjects who had large responses to the load showed almost the same negative correlation (both having smaller than -0.95 ) in comparison with the frequency analysis. In addition, the Wilcoxon signed-rank test was also performed because the paired t-test assumes a normal distribution of the data. The results of the simple correlation also showed statistically significant differences between the respiratory-phase domain and the frequency domain $(P<0.05)$, whereas the rank correlation result cannot be tested because of the data loss by the same correlation coefficient. In summary, these results demonstrate that the respiratory-phase domain analysis had a more effective response to the load than the conventional frequency analysis, and we can conclude this former method could evaluate MWL accurately.

\subsection{Short-term Extraction of RSA}

In this study, the entire $300 \mathrm{~s}$ was used for analysis of RSA; however, short-term analysis can be adapted using moving-averaging. Thus, short-term extraction of RSA was attempted. In general, the accuracy and averaging range have a trade-off relationship, and the optimal range must be decided by the relationship between variation and needed accuracy. To determine the number of respirations for the averaging window, we needed data for a long-term RSA time series in the same condition. For this purpose, the RSAs of seven healthy males were taken at rest and in a sitting posture for $30 \mathrm{~min}$. The amplitude of RSA in this data had an anti- 
A

(a)

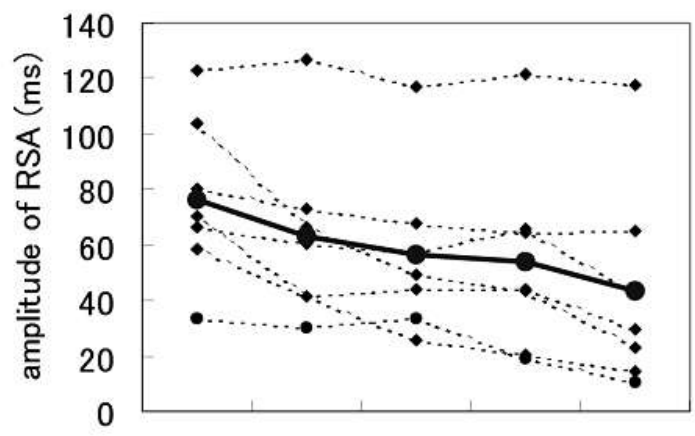

Rest Task1 Task2 Task3 Task4

B

(a)

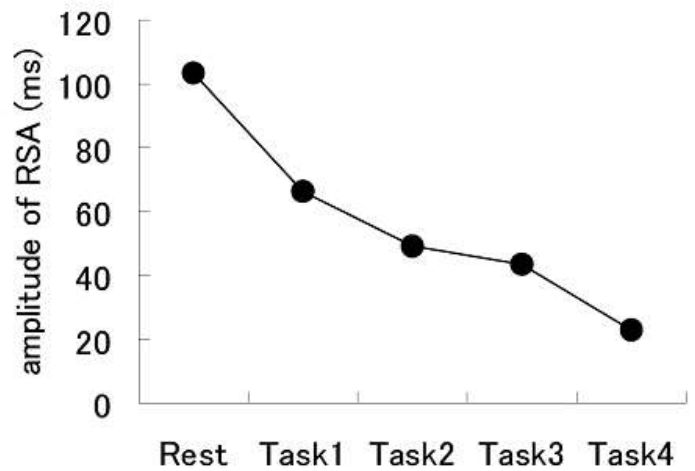

(a)

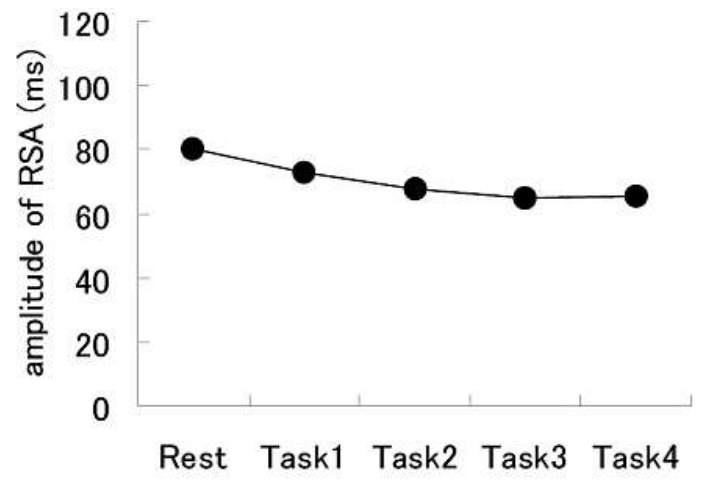

(b)

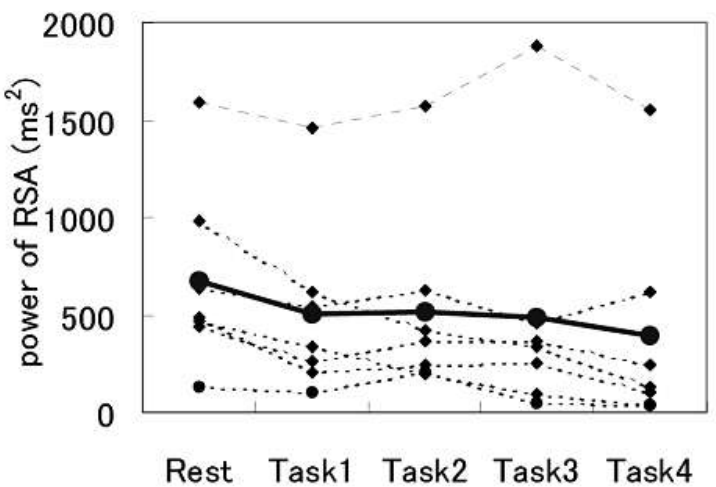

(b)

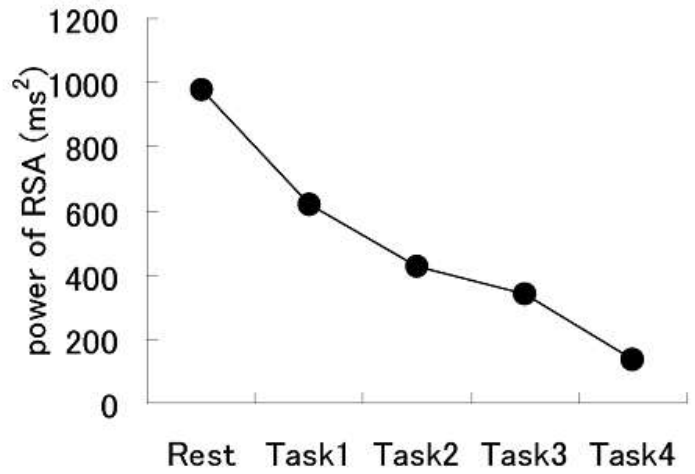

(b)

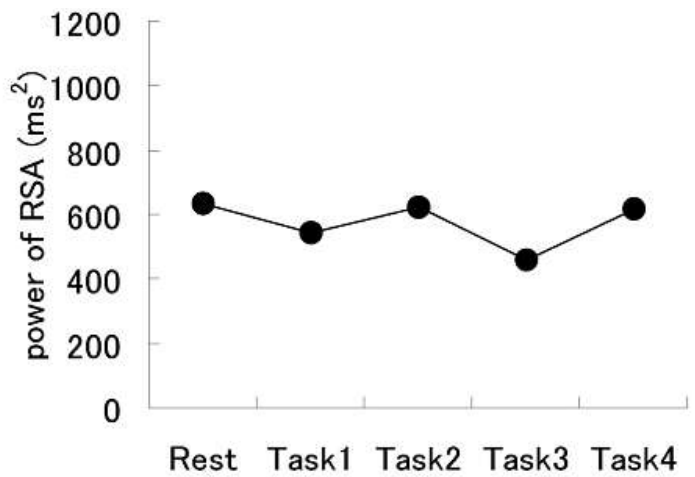

Fig. 6 Results of the amplitude and power of RSA as determined by the two methods: (a) the amplitude of RSA determined by respiratory-phase domain analysis, (b) power of RSA determined by frequency domain analysis (FFT). A: Data of all subjects. Solid line represents mean value and dotted lines represent individual values; B: Data from one subject who had a large response against the task; $C$ : Data from one subject who had a small response against the task. The results of the two analyses ((a) and (b)) are alike in subject of $B$, while they differ in subject of $C$. correlation because of the MWSA, and had a standard deviation of $32.1 \mathrm{~ms}$, which is the mean of the standard deviation of RSA amplitude with each respiration. With regard to the mental arithmetic task, the difference of the amplitude of RSA between a weak load
(Task 2: the averaged NASA-TLX score, 45.6) and a strong load (Task 4: 68.9) was $13.1 \mathrm{~ms}$. A normal distribution and the independence of the amplitude of RSA were roughly supposed, and 24 respirations were needed to distinguish these two tasks with the safety ratio of twice of the standard deviation (i.e. estimated standard deviation after averaging was within half of $13.1 \mathrm{~ms}$ ). Figure 7 shows the result of the moving average of 24 respirations (including 11 respirations before and 12 respirations after the actual 
Table 1 Correlation coefficients between the MWL and the amplitude of RSA as determined by respiratory-phase domain analysis and frequency domain analysis: the simple correlation and the rank correlation between the amplitude or power of RSA and the load level. The group-wise results are also shown in this table. A subject was categorized in the large response group if his amplitude of RSA in Task 4 was less than one-fourth of what was in rest. Data for all subjects are mean values with standard deviations. ${ }^{*}$ indicates a statistically significant difference between the respiratory-phase domain and the frequency domain $(P<0.05)$.

\begin{tabular}{|l|l|l|l|l|}
\hline & \multicolumn{2}{|l|}{ Simple correlation } & \multicolumn{2}{l|}{ Rank correlation } \\
\hline & $\begin{array}{l}\text { Respiratory-phase } \\
\text { domain }\end{array}$ & $\begin{array}{l}\text { Frequency } \\
\text { domain }\end{array}$ & $\begin{array}{l}\text { Respiratory-phase } \\
\text { domain }\end{array}$ & $\begin{array}{l}\text { Frequency } \\
\text { domain }\end{array}$ \\
\hline Small response group $(n=5)$ & -0.79 & -0.37 & -0.74 & -0.48 \\
\hline Large response group $(n=2)$ & -0.96 & -0.98 & -1.0 & -1.0 \\
\hline All subjects $(n=7)$ & $-0.84 \pm 0.14^{*}$ & $-0.54 \pm 0.47$ & $-0.81 \pm 0.18^{*}$ & $-0.63 \pm 0.34$ \\
\hline
\end{tabular}

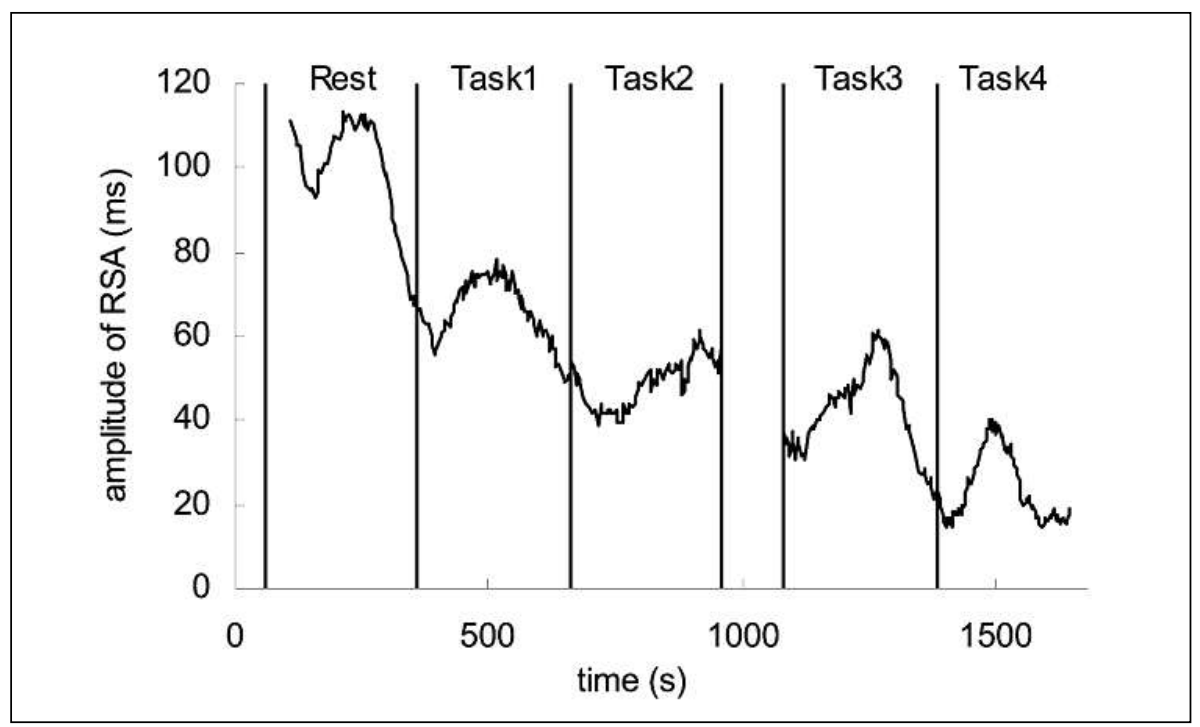

Fig. 7 Short-term extraction of the amplitude of RSA determined by the respiratory-phase domain analysis in one subject, who is the same as that described in Fig. 6B.

time step) in the subject who had the largest reaction to the load. In this case, the amplitude of RSA decreased at the beginning of all the sessions and it was supposed that this subject had difficulty adapting to the changing speed of the task. This method can detect such a partial change of RSA amplitude.

\section{Discussion}

\subsection{Evaluation of Mental Workload}

In this study, three indices of MWL were extracted. The subjective index, the NASA- the amplitude of RSA decreased during a mental arithmetic task, a finding that is consistent with the previous studies on MWL $[2,6]$ or mental stress [17]. Moreover, the physiological index might account for the inter-independent differences caused by biological rhythm or physical condition.

Considering the level of the load in detail, we divided the quality of the task into three factors, namely the difficulty of the task, the amount of work, and the time pressure. In this experiment, the difficulty of the task remained the same, and the amount of work (i.e., the number of questions) increased linearly, while the time pressure of the task did not always increase linearly. But the time pressure of the task did not increase abruptly because the average response time in Task 1, which had the longest interval, was $2.96 \mathrm{~s}$ and did not significantly exceed the interval in Task 4 (3 s). However, the vagal activity against MWL or subjective feelings is thought to be a complex, nonlinear function, and this nonlinearity might cause the decrease of the negativity of the simple correlation between the level of load and the amplitude of RSA. Further experiments with various degrees of tasks are needed to further explore this point. In addition, the experimental conditions of this study were not suitable for the evaluation of short-term extraction of RSA, because the task continued for $300 \mathrm{~s}$ at the same speed. Another experiment involving many short-term tasks is needed to investigate the precise relationship between the short-term extraction of RSA and the load level (or other indices).

Furthermore, the correlation between other physiological valuables and the amplitude of RSA by the proposed method or the load level was investigated. At first, the mean RRI during each task was calculated and the correlation coefficient against the amplitude of RSA was $0.72 \pm 0.34$. The difference between the mean RRI and the amplitude of RSA is known to be mainly caused by sympathetic nervous activity to heart. Therefore, these correlations would depend on the types of task. Further investigation is needed about how the types of task contribute to the correlation between the mean RRI and the amplitude of RSA. Also, the correlation coefficient was calculated 
against the load level and it was $-0.74 \pm$ 0.33 . This value was better than the frequency analysis and worse than the proposed method. Secondary, the same analysis was performed with 1) amplitude and 2) interval of respiration. 1) The correlation coefficient against the amplitude of RSA was $0.46 \pm$ 0.65 , while that against the load level was $-0.35 \pm 0.61 .2)$ The correlation coefficient against the amplitude of RSA was $0.81 \pm$ 0.27 , while that against the load level was $-0.79 \pm 0.25$. These results indicate that the changes of RSA of this experiment were to some extent assisted by the changes of respiration, which was also brought by autonomic nervous activity.

\subsection{Consideration of Signal Processing}

The analysis of the respiratory-phase domain was compared with frequency analysis by using two methods; i.e., the simple correlation and the rank correlation against the load level. The simple correlation requires the assumption that the RSA amplitude linearly responded to the load level. Otherwise, the rank correlation only requires an assumption that the RSA amplitude decreased monotonously as the load level increased, which seems to be a more reliable assumption. Also, a strength of this assumption is that it is not affected by the unit of the RSA such as amplitude, power, or logarithm of power. But this evaluation involves the risk that even a small error can change the ranking and produce a large difference in the correlation coefficient. Therefore, it is necessary to consider both results. The results of both cases show that the respiratory-phase domain analysis had a higher negative correlation and a smaller standard deviation. These results show that the respiratoryphase domain analysis followed the load level more closely and had the greater stability as an index than did frequency analysis. It is considered that if there is a sufficiently large reaction to the load, the correlation coefficients of both analyses converge to -1 with no difference. Therefore, it is very important that the data for the subject who has a weak correlation against the task are improved by the respiratory-phase domain analysis, as shown in Table 1 (small response group).

The question of why the respiratoryphase domain analysis is effective in comparison with the frequency analysis was considered. The amplitude of the RSA determined by the proposed method is interchangeable with the extraction using a Fourier transform. In the case of paced breathing, which is widely used in physiological experiments for the accurate extraction of RSA in the frequency domain $[3,18]$, the frequency of RSA turns out to be a constant value that corresponds to respiratory frequency. Furthermore, respiratoryphase domain analysis has two strengths in comparison with frequency analysis: a) the respiratory-phase domain analysis can use data effectively, while the Fourier transform produces errors because of the window function or harmonics, or because the FFT cannot use the full data-length equally if the data-length is not $2^{n}$; and $b$ ) the procedure of data selection improves the accuracy of the extraction. In this study, the correlation coefficient of frequency analysis has a very small negative correlation compared with the respiratory-phase domain analysis. There could be two reasons for this. One is the large tachycardia induced by swallowing, and the other is the low frequency respiration, i.e., less than $0.15 \mathrm{~Hz}$. For swallowing, the induced tachycardia was mixed with the power more than $0.15 \mathrm{~Hz}$ in the frequency analysis, and the influence was mainly observed in the data of subjects whose results for the correlation coefficient of frequency analysis did not have large negative values. For low frequency respiration, this influence was not observed clearly in this experiment, but we feared that the power of RSA could be underestimated in the frequency analysis. Theoretically, if there are low frequency respirations of less than $0.15 \mathrm{~Hz}$, the power of RSA reaches a level outside of its calculation range and it is equivalent to there being no RSA in the period of low frequency respiration. These tendencies show that the conventional analysis does not produce a high level of accuracy because of noise or improper respiration, and the tendencies are large enough to have statistical significance in a real situation. In this experiment, a mental arithmetic task was adopted because the subject was able to sit quietly, and unexpected events were prevented. However, in an actual work context or a situation in which there exist many noises (e.g., coughs, sneezes, speaking, extrasystoles, or sensory errors), the respiratory-phase domain analysis will be more effective because the data that are distorted by noise can be efficiently removed.

The accuracy of the estimation of the cardiac vagal activity can be improved by improving the procedure of data selection. Removing the data that contains physiological influences that are known to generate temporal tachycardia and/or bradycardia would improve the accuracy of the results. In addition, removing the data that has statistically different waveforms, which was recently proposed by Gilad [19], would also be effective. Furthermore, it would be useful to modify the physiological models [20-22] for the respiratory-phase domain and to estimate the parameter of the model. These improvements could provide a better understanding of RSA.

In a short-term analysis, Gianaros et al. [23] investigated the transition of RSA by means of frequency analysis of continuous $1 \mathrm{~min}$ data in an experiment on motion sickness severity. To investigate such a time transition, the short-term extraction of RSA that we proposed in Section 3.3 is more effective because the signal to noise ratio is low in the short-term data. In addition, it is important that the proposed method is performed in real-time and with the task well controlled. Further improvement of the signal-processing algorithm is needed for this purpose.

Some studies focusing on the relationship between RSA and respiratory information have been performed. Yli-Hankala et al. [24] showed a response waveform by the periodic input of positive pressure ventilation, and Gilad et al. [19] also showed the waveform of RSA by using the onset of the expiration as a trigger. Wang et al. [25] defined the vector of the RSA by plotting the $\mathrm{R}$-wave and the phase of the respiration that is triggered by the onset of the inspiration. There were three main differences between the proposed method and these previous methods. First, with the use of the DCSI method, a continuous and non-delayed ef- 
fect can be analyzed. Second, the phase of respiration was calculated for accurate extraction of the RSA waveform. This procedure is effective because respiration is a nonlinear oscillation. Third, the data of each breath was selected and removed when it did not reflect vagal activity in the physiological sense. Though these differences reveal precise information about the waveform of the RSA, calculation of the phase of respiration is relatively sensitive. If 0 is not crossed in the period of inspiration and expiration, the respiratory phase does not increase $2 \pi \mathrm{rad}$ at that respiration. By filtering, the respiratory phase was successfully extracted in this experimental condition because the subject was able to sit quietly during the mental arithmetic task. But in the case of another experimental condition, to amplify the small respiration or to use different sensors might be necessary to accurately determine the respiratory phase.

\subsection{Application of the Proposed Method}

RSA is a complex phenomenon because the respiration and vagal activity themselves are complex. The respiratory-phase domain analysis is effective for extracting RSA and investigating its underlying mechanisms. By extracting the RSA accurately, much information about the complex function of vagal activity can be obtained. Also, HRV is a nonlinear and complex fluctuation and the proposed method is also effective for decomposing HRV into RSA and other components. It is, therefore, possible to investigate the effect of RSA on the complexity of the HRV in the statistical physical analysis, a type of analysis that has recently attracted interest $[8,9]$.

Furthermore, the reaction of cardiac vagal activity to external and daily situations, including emotional changes [26], is important for maintaining homeostasis, but a great deal about this remains unknown. The proposed method is convenient enough to extend the measurement to daily situations, and the measurement of physiological parameters in daily situations is our next target.

\section{Conclusion}

In this study, we proposed a simple and effective method for extracting the amplitude of RSA in the respiratory-phase domain. RSA was extracted in an experiment involving a mental arithmetic task along with subjective and objective indices. The results show that under the appropriate task, the amplitude of RSA decreased as the load level increased, and the proposed method was demonstrated to effectively evaluate MWL. Furthermore, a comparison of the proposed method with conventional frequency analysis showed that respiratoryphase domain analysis followed the load level more accurately than did the frequency analysis. These results confirm that the proposed method can effectively estimate cardiac vagal activity and is able to evaluate the MWL of workers with a high level of accuracy.

\section{Acknowledgments}

The authors wish to thank K. Furukawa for supporting the experiment and Dr. M. Imran Al-Haq for improving the manuscript. This research is partly supported by the 21st century COE program "Information Science and Technology Strategic Core" from the Ministry of Education, Culture, Sports, Science and Technology, Japan.

\section{References}

1. Task Force of the European Society of Cardiology and the North American Society of Pacing and Electrophysiology. Heart rate variability: standards of measurement, physiological interpretation, and clinical use. Circulation 1996; 93 : 1043-65.

2. Allen MT, Crowell MD. Patterns of autonomic response during laboratory stressors. Psychophysiology 1989; 26: 603-14.

3. Hayano J, Mukai S, Sakakibara M, Okada A, Takata K, Fujinami T. Effect of respiratory interval on vagal modulation of heart rate. Am J Physiol 1994; 267: H33-H40.

4. Katona PG., Jih F. Respiratory sinus arrhythmia: noninvasive measure of parasympathetic cardiac control. J Appl Physiol 1975; 39: 801-5.

5. Pomeranz B, Macaulay RJB, Caudill MA, Kutz I, Adam D, Gordon D, Kilborn KM, Barger AC, Shannon DC, Cohen RJ, Benson H. Assessment of autonomic function in humans by heart rate spectral analysis. Am J Physiol 1985; 248: H151-3.

6. Hoshikawa Y, Yamamoto Y. Effects of stroop color word conflict test on the autonomic ner- vous system responses. Am J Physiol 1997; 272 : H1113-21.

7. Suzuki S, Toyabe S, Moroda T, Tada T, Tsukahara A, Iiai T, Minagawa M, Maruyama S, Hatakeyama K, Endoh K, Abo T. Circadian rhythm of leucocytes and lymphocyte subsets and its possible correlation with the function of the autonomic nervous system. Clin. Exp. Immunol 1997; 110: 500-8.

8. Ivanov PC, Amaral LAN, Goldberger AL, Havlin S, Rosenblum MG, Struzik ZR, Stanley HE. Multifractality in human heartbeat dynamics. Nature 1999; 399: 461-5.

9. Kiyono K, Struzik ZR, Aoyagi N, Sakata S, Hayano J, Yamamoto Y. Critical scale-invariance in healthy human heart rate. Phys Rev Lett 2004; 93 : 178103-1-4.

10. Kotani K, Hidaka I, Yamamoto Y, Ozono S. Analysis of respiratory sinus arrhythmia with respect to respiratory phase. Methods Inf Med 2000; 39: 153-6.

11. Svensson E, Angelborg-Thanderz ML, Sjöberg. Mission challenge, mental workload and performance in military aviation. Aviat Space Environ Med 1993; 64: 985-91.

12. Hart SG, Staveland LE. Development of NASATLX (Task Load Index): results of empirical and theoretical research. Human Mental Workload, Elsevier Science Publishers; 1988: pp 139-83.

13. Han K, Nagel JH, Schneiderman N. A continuous representation of heart rate. Proc Ann Int Conf IEEE/EMBS 1992; 14: 785-6.

14. Schäfer C, Rosenblum MG, Abel H-H, Kurths J. Synchronization in the human cardiorespiratory system. Phys Rev E 1999; 60: 857-70.

15. Baumert M, Baier V, Haueisen J, Wessel N, Meyerfeldt U, Voss A. Forecasting of life threatening arrhythmias using the compression entropy of heart rate. Methods Inf Med 2004; 43: 202-6.

16. Mainardi LT, Montano N, Cerutti S. Automatic decomposition of Wigner distribution and its application to heart rate variability. Methods Inf Med 2004; 43: 17-21.

17. Lucini D, Norbiato G, Clerici M, Pagani M. Hemodynamic and autonomic adjustments to real life stress conditions in humans. Hypertension 2002; 39: 184-8.

18. Hayano J, Sakakibara Y, Yamada A, Yamada M, Mukai S, Fujinami T, Yokoyama K, Watanabe Y, Takata K. Accuracy of assessment of cardiac vagal tone by heart rate variability in normal subjects. Am J Cardiol 1991; 67: 199-204.

19. Gilad O, Swenne CA, Davrath LR, Akselrod S. Phase-averaged characterization of respiratory sinus arrhythmia pattern. Am J Physiol Heart Circ Physiol 2005; 288: 504-10.

20. DeBoer RW, Karemaker JM, Strackee J. Hemodynamic fluctuation and baroreflex sensitivity in humans: a beat-to-beat model. Am J Physiol 1987; 253: H680-9.

21. Kotani K, Takamasu K, Ashkenazy Y, Stanley HE, Yamamoto Y. Model for cardio-respiratory synchronization in humans. Phys Rev E 2002; 65: 051923-1-9. 
22. Pyetan E, Akselrod S. A theoretical appraisal of the dependence of respiratory sinus arrhythmia on gradual vagal blockade. Methods Inf Med 2004; 43: $52-5$.

23. Gianaros PJ, Quigley KS, Muth ER, Levine ME, Vasko RC Jr, Stern RM. Relationship between temporal changes in cardiac parasympathetic activity and motion sickness severity. Psychophysiology 2003; 40: 39-44.
24. Yli-Hankala A, Porkkala T, Kaukinen S, Häkkinen V, Jäntti V. Respiratory sinus arrhythmia is reversed during positive pressure ventilation. Acta Physiol Scand 1991; 141: 399-407

25. Wang DY, Pomfrett CJD, Healy TEJ. Respiratory sinus arrhythmia: a new, objective sedation score. British Journal of Anaesthesia 1993; 71: 354-8.

26. Watkins LL, Grossman P, Krishnan R, Sherwood A. Anxiety and vagal control of heart rate. Psychosom Med 1998; 60: 498-502.

\section{Correspondence to:}

Kiyoshi Kotani

Graduate School of Information Science and Technology The University of Tokyo

7-3-1 Hongo, Bunkyo-ku Tokyo 113-8656

Japan

E-mail: kota@nano.pe.u-tokyo.ac.jp 